\title{
IMPLEMENTATION OF THE FAST RF FEEDBACK ON THE DAФNE BEAM LONGITUDINAL DYNAMICS SIMULATION CODE
}

\author{
A. Gallo, F. Marcellini, M. Migliorati
}

\section{Introduction.}

As reported in other papers ${ }^{1,2}$, multibunch instabilities in DAФNE have been studied by means of a dedicated simulation code describing the beam-cavity HOM interaction in the time domain. The radiation damping effect and the bunch-bybunch feedback system aimed at curing the multibunch instabilities were also included in the code.

On the other hand the interaction with the cavity fundamental mode, that strongly affects the beam longitudinal dynamics ${ }^{3,4}$, has been so far neglected in the code. A pure sinusoidal accelerating voltage independent from beam synchrotron oscillations was assumed in the simulations, loosing therefore all the information on beam loading effects. This ideal assumption is the so called "perfectly compensated case" and corresponds to an infinite gain RF feedback loop closed around the accelerating cavity.

Recently the code has been modified to include the effect of a real RF feedback whose gain is limited by the total group delay of the loop ${ }^{5}$. Updated simulations including the beam loading effects are reported in this note, together with a general description of the model adopted to implement the feedback network in the code.

\section{General approach to the RF feedback implementation.}

The typical schematics and the corresponding block diagram of an RF feedback aimed at reducing the cavity dynamical impedance are shown in Fig. 1. The L(s) block represents the transfer function of the whole loop components but the cavity, that has its own transfer function $\mathrm{C}(\mathrm{s})$ equal to:

$$
C(s)=\frac{2 \beta}{\beta+1} \frac{\frac{s}{Q_{L} \omega_{c}}}{\frac{s^{2}}{\omega_{c}{ }^{2}}+\frac{s}{Q_{L} \omega_{c}}+1}
$$

where $\omega_{c}$ is the cavity resonant angular frequency, $\beta$ is the cavity-generator coupling factor and $\mathrm{Q}_{\mathrm{L}}=\mathrm{Q}_{\mathrm{O}} /(1+\beta)$ is the loaded cavity quality factor. 
The cavity fundamental mode dynamical impedance $Z(s)$ is reduced by the feedback response down to:

$$
Z(s)=\frac{Z_{o}(s)}{1+L(s) C(s)}
$$

where

$$
Z_{o}(s)=\frac{R}{Q} \frac{\frac{s}{\omega_{c}}}{\frac{s^{2}}{\omega_{c}^{2}}+\frac{s}{Q_{L} \omega_{c}}+1}
$$

is the fundamental mode impedance without feedback.
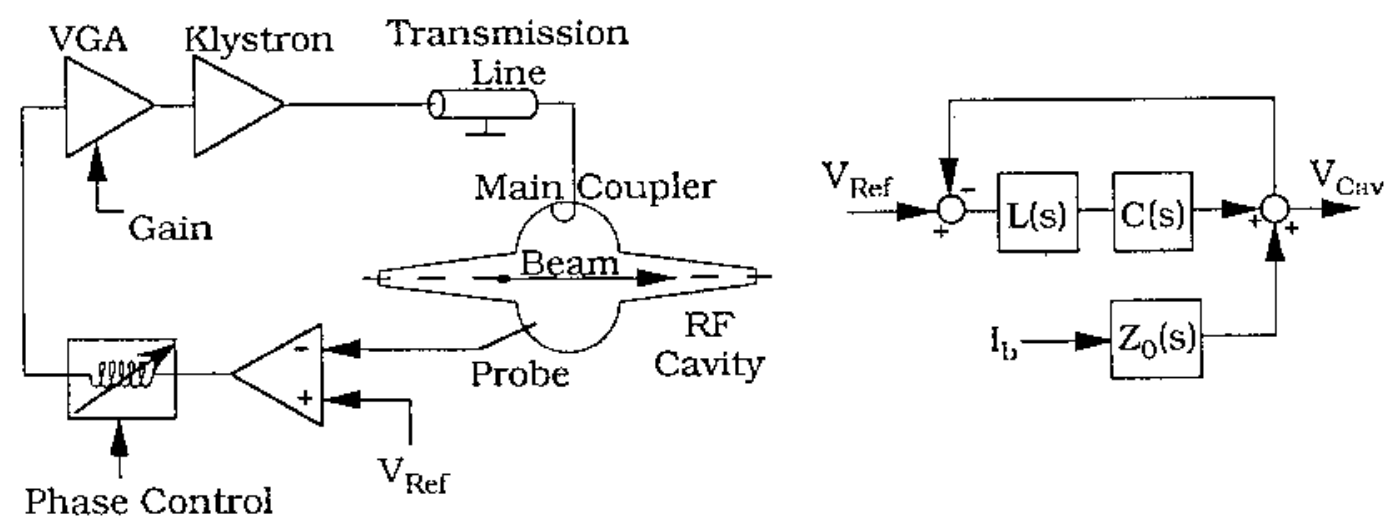

Fig. 1: RF feedback system and block diagram.

In order to implement the RF feedback operation in the simulation code a circuit model of the L(s) block must be chosen. The time evolution of the system can then be studied with the superposition theorem considering the terms due to the RF reference generator and to the beam separately.

The RF reference generator gives a sinusoidal regime term of angular frequency $\omega_{\mathrm{r}}$ to the cavity accelerating voltage. The beam contributes to the accelerating voltage by exciting free oscillations in the system at each bunch passage. The status of the system, while executing free oscillations is determined at any time by a set of conjugated variables representing the capacitance voltages and the inductance currents of the whole feedback network. The number of these status variables depends on the order of the open loop transfer function $\mathrm{H}(\mathrm{s})=\mathrm{L}(\mathrm{s}) \mathrm{C}(\mathrm{s})$. At any new bunch passage the status of the system, together with the kick given by the bunch, is summarized in a set of new initial conditions which determines the free oscillation evolution. The time evolution of the status variables is determined by a transport matrix according to:

$$
\mathbf{X}\left(t_{o}+t\right)=\mathbf{A}(t) \mathbf{X}\left(t_{o}\right)
$$

where $\mathbf{X}$ is the vector of the conjugated variables, $\mathbf{A}$ is the transport matrix and $t_{0}$ is the starting time, i.e., the time of the bunch passage.

The matrix elements $a_{i j}(t)$ are obtained from the Laplace inverse transform of the transfer functions $a_{i j}(s)$ which correlate each status variable with the other ones. 
Since these transfer functions refer to a negative feedback network, they are of the following general form:

$$
a_{i j}(s)=\frac{a^{\prime}{ }_{i j}(s)}{1+L(s) C(s)}
$$

and can be computed by solving the network with standard techniques.

The choice of a realistic model for the L(s) block is of crucial importance because the associated frequency response limits the maximum attainable feedback gain and consequently the feedback efficiency. On the other hand, a too complex model produces a too large transport matrix and the system solution may get very unpractical.

\section{The RF feedback lumped circuit model.}

The major contributions to be included in the response L(s) are the klystron frequency response and the delay of the lines used for the loop connections.

The frequency response of the DAФNE klystron TTE 2145 has been measured during the factory acceptance test of the tube. The result reported in Fig. 2 shows that the frequency response can be very well fitted by a double resonator model.

The total delay of the cables and lines used for the feedback connections is $\mathrm{T}_{\mathrm{O}} \approx 100 \mathrm{nsec}$ corresponding to a total length of about $30 \mathrm{~m}$. To include both contributions in L(s) we should consider a fourth-order transfer function multiplied by the pure delay term $\exp \left(-\mathrm{s} \mathrm{T}_{\mathrm{o}}\right)$. The resulting transfer function is too complex to be handled in the previously described formalism.

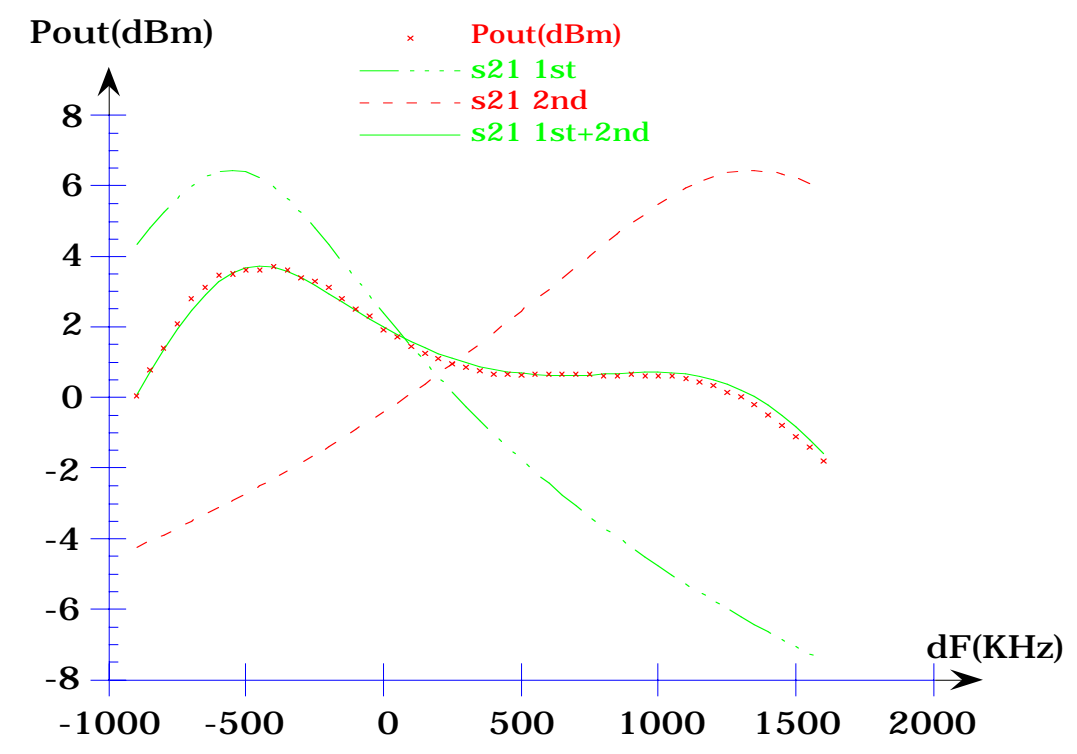

Fig. 2: Frequency response of the DAФNE klystron fitted by a double resonator model

In order to simplify the model, the klystron response around the operating frequency can be approximated with an additional pure delay term $\exp \left(-\mathrm{sT}_{\mathrm{k}}\right)$ where $\mathrm{T}_{\mathrm{k}}$ is the klystron group delay, i.e. the derivative of the output phase with respect to the angular frequency of the excitation. 
A group delay $\mathrm{T}_{\mathrm{k}} \approx 160$ nsec has been measured during the factory acceptance tests of the klystron TH 2145.

With this last assumption the L(s) transfer function becomes a pure delay term of the following form:

$$
L(s)=G \exp \left(-s T_{d}\right)=G \exp \left(-s T_{o}-s T_{k}\right)
$$

where the total delay $T_{d}$ is less than 300 nsec.

In spite of the simplicity of this last expression, the presence of an exponential term in the open loop transfer function complicates a lot the computation of the Laplace inverse transform of the $a_{i j}(s)$ functions ${ }^{6}$. To simplify the inverse transform operation it is possible to well approximate a pure delay term with a special lumped network. Let us consider the following expression for L(s):

$$
L(s)=-G \frac{\frac{s^{2}}{\omega_{d}^{2}}-4 \frac{s}{\omega_{d}^{2} T_{d}}+1}{\frac{s^{2}}{\omega_{d}^{2}}+4 \frac{s}{\omega_{d}^{2} T_{d}}+1}
$$

corresponding to the frequency response of the lumped network in Fig. 3, assuming $\mathrm{T}_{\mathrm{d}}=4 \mathrm{R}_{\mathrm{d}} \mathrm{C}_{\mathrm{d}}$ and $\omega_{\mathrm{d}}=\left(\mathrm{L}_{\mathrm{d}} \mathrm{C}_{\mathrm{d}}\right)^{-1 / 2}$. As in the case of a pure delay line, the amplitude of this transfer function is equal to 1 at any frequency, while the phase response is shown in Fig. 4 compared to the linear phase advance of the pure delay line. The linearity is very good in the $\pm \pi / 2$ range, with a total phase response range of $\pm \pi$.

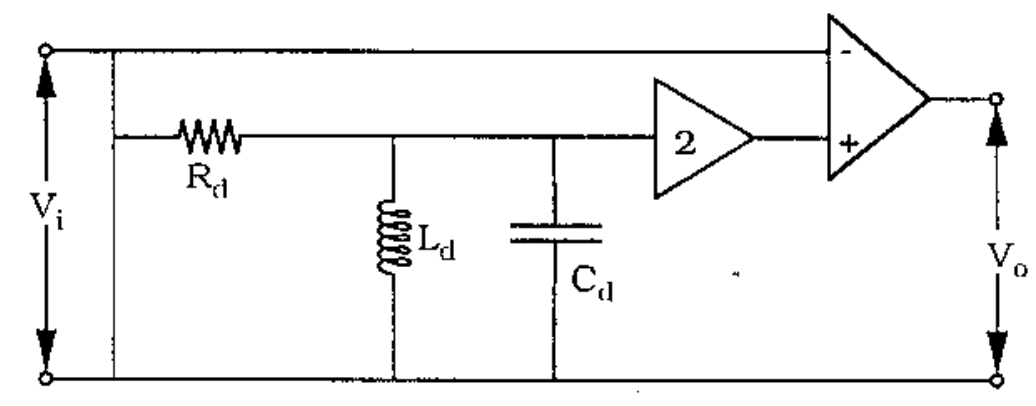

Fig. 3: Lumped network used for the RF feedback system.

In order to preserve the negative sign of the feedback and to exploit the linear part of the frequency response, the network parameters must be such that $\omega_{\mathrm{d}} \approx \omega_{\mathrm{c}}$.

By adopting the model of Fig. 3, the total open loop transfer function $H(s)=L(s)$ $\mathrm{C}(\mathrm{s})$ is of the fourth-order. Then the time evolution of the system is determined by a $4 \times 4$ transport matrix which can be computed with a reasonable effort, as shown in Appendix 1.

Under the assumption (7) the $\mathrm{H}(\mathrm{s})$ phase response range is wider than $\pm \pi$ and an excessive open loop gain in the simulation leads the feedback system to instability reproducing the actual system behavior. This confirms that the lumped delay network is a realistic model. 


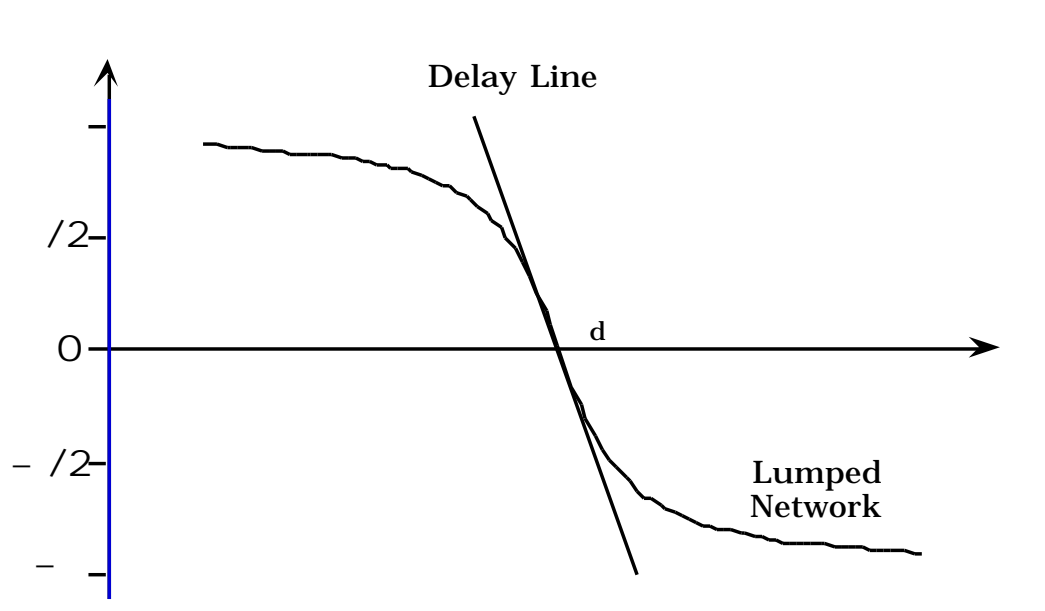

Fig. 4: Phase response of the lumped network used in the simulation code.

The complete circuit model of the RF feedback including the cavity is shown in Fig. 5.

The four status variables are the two voltages across the capacitances $C_{c}$ and $C_{d}$ and the two currents flowing through the inductances $\mathrm{L}_{\mathrm{c}}$ and $\mathrm{L}_{\mathrm{d}}$. The beam is represented by a Dirac- $\delta$ current generator that suddenly charges the cavity capacitance. Other 4 Dirac- $\delta$ generators have been introduced in the model to force the initial conditions at every bunch passage.

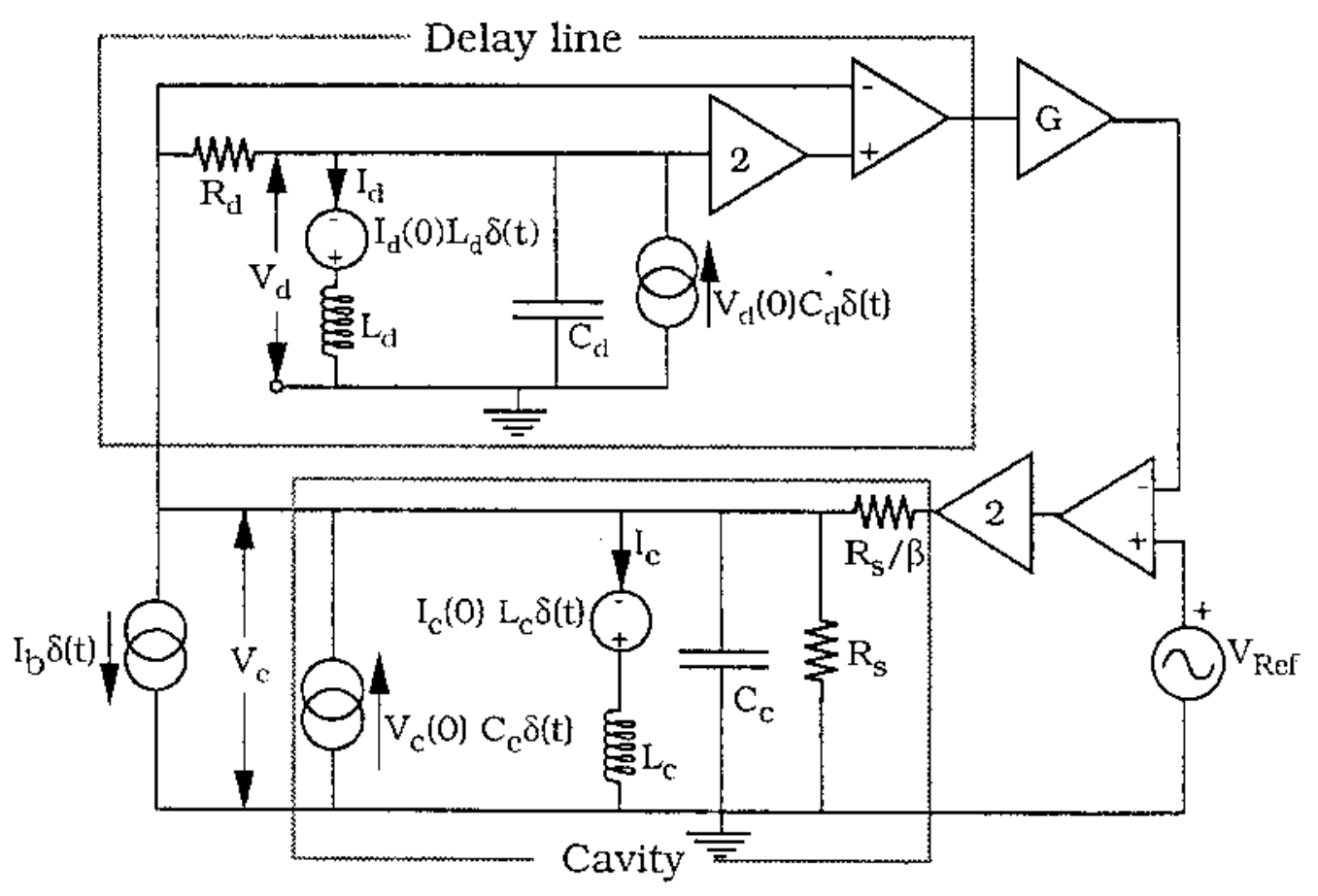

Fig. 5: Circuit model of the RF feedback. 


\section{The transfer function and the stability conditions.}

A linear feedback system is stable as long as its open loop transfer function does not enclose the $(-1,0)$ point in the polar diagram (Nyquist criterion).

In practice a real system can not operate safetly if it is too close to the instability threshold. A commonly used parameter to indicate how close the system is to the instability point is the so called "phase margin" $\phi_{m}$ which is the open loop transfer function phase with respect to the negative abscissa at the frequency where the amplitude is equal to 1 .

The polar diagram of the open loop transfer function $\mathrm{H}(\mathrm{s})$ obtained by multiplying expressions (1) and (7) is shown in Fig. 6. A phase margin of $45^{\circ}$ is obtained with a maximum gain of about 30 for a 300 nsec total group delay. The plot of the cavity fundamental mode impedance, as given by (2), for four phase margin values is shown in Fig. 7.
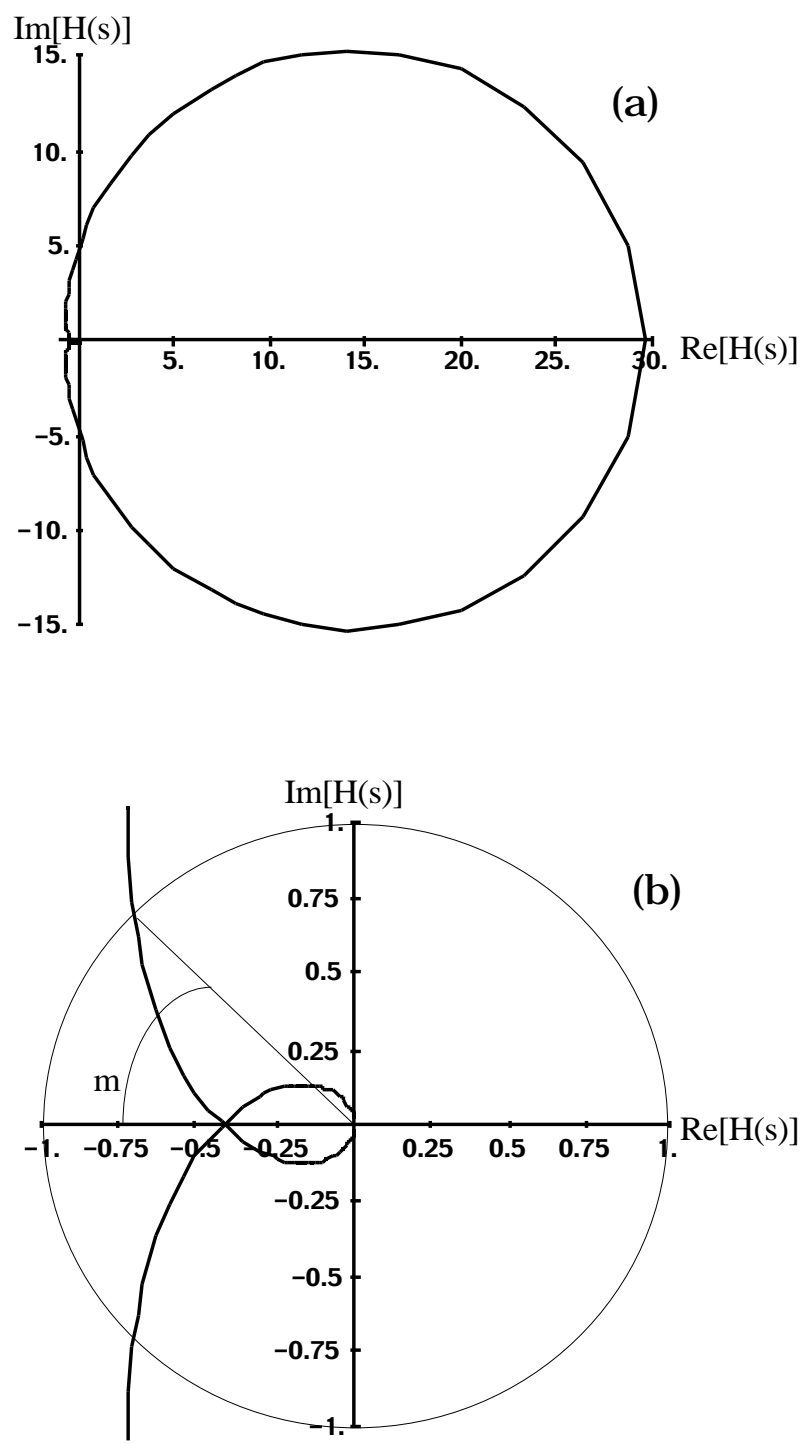

Fig. 6: (a) Polar diagram of the open transfer function.

(b) Expanded view of (a) around the origin. 


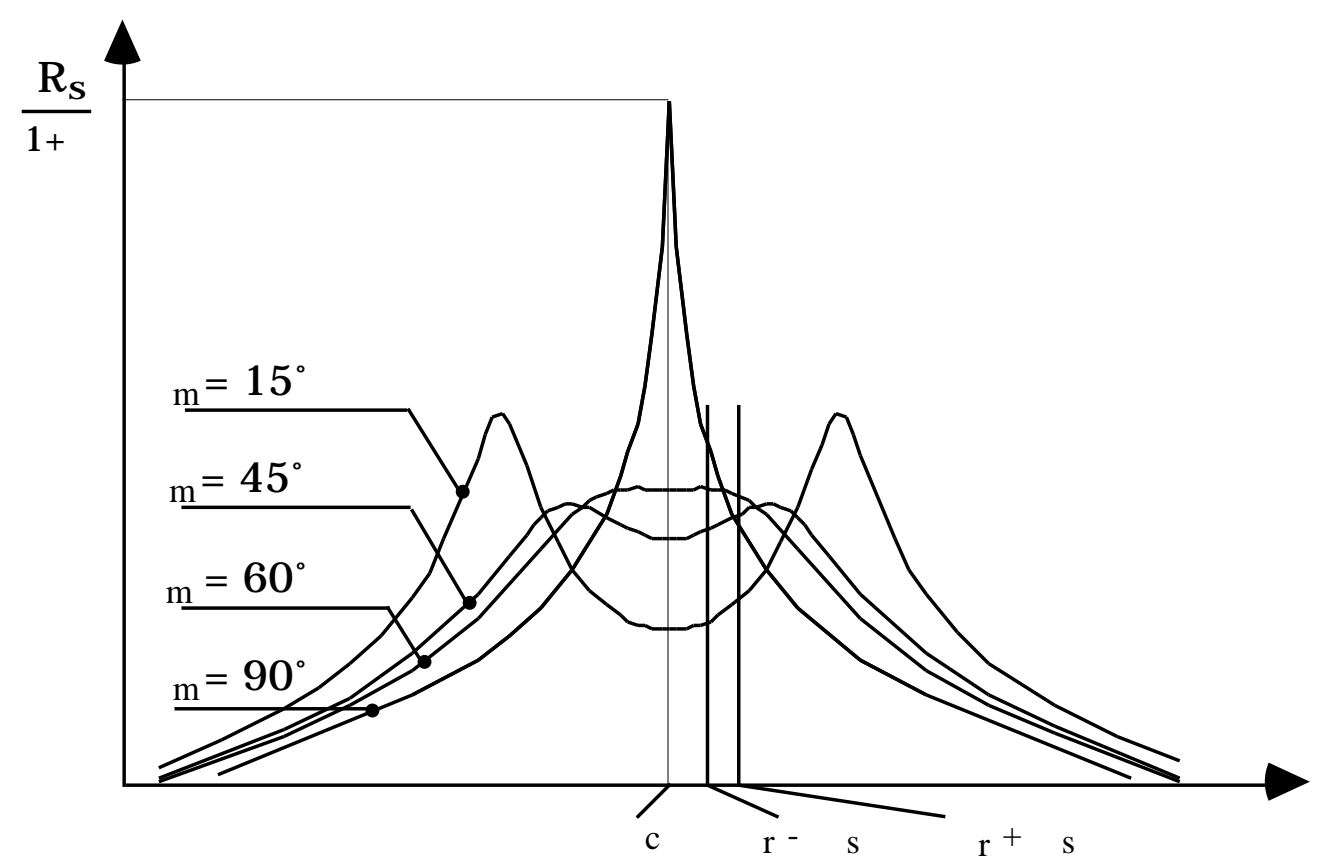

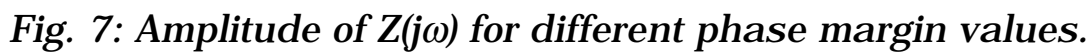

\section{Simulation code tests.}

In order to verify the correctness of the simulation code, we have investigated some particular situations useful to tune the values of the RF feedback parameters.

\subsection{Dynamic Robinson's instability.}

When the phase margin of the feedback system is between $0^{\circ}$ and $60^{\circ}$, the fundamental mode impedance is deformed in such a way that, for the usual positive detuning values of the cavity $\left(\Delta \omega=\omega_{\mathrm{r}}-\omega_{\mathrm{c}}>0\right)$, the beam is unstable (dynamic Robinson's instability ${ }^{7}$ ). This is because the stable synchrotron line of the multibunch "0" oscillation mode is lower than the unstable line, as shown in Fig. 7. On the contrary, when the phase margin is larger than $60^{\circ}$, the beam is stable.

From the single particle synchrotron equation it is possible to obtain analytically the rise time of the dynamic Robinson's instability. In fact by neglecting the radiation damping effect and considering the beam loading voltage, we can write ${ }^{7}$ :

$$
\ddot{\varphi}+\frac{1}{\tau_{r}} \dot{\varphi}+\omega_{s}^{2} \varphi=0
$$

where $\varphi$ is the phase shift of the particle with respect to the synchronous phase, $\omega_{\mathrm{S}}$ is the synchrotron angular frequency and the rise time $\tau_{\mathrm{r}}$ is given by:

$$
\frac{1}{\tau_{r}}=\frac{I_{b} \alpha_{c} \omega_{r}}{\omega_{s}\left(E_{o} / e\right) T}\left\{\operatorname{Re}\left[Z\left(j\left(\omega_{r}+\omega_{s}\right)\right)-Z\left(j\left(\omega_{r}-\omega_{s}\right)\right)\right]\right\}
$$

with $\alpha_{c}$ the momentum compaction, $E_{o}$ the nominal energy, and $\mathrm{T}$ the revolution period. 
The rise time calculated with eq. (9) is in excellent agreement with that obtained by fitting the simulation code output.

\subsection{Static Robinson's stability limit.}

Let us define $\phi_{\mathrm{L}}$ as the phase of the load (cavity impedance and beam) seen from the cavity main coupler. When $\phi_{\mathrm{L}}=0$ this impedance is purely resistive.

The static Robinson's stability limit ${ }^{8}$ relates the maximum current storable in the ring to $\phi_{\mathrm{L}}$. In Fig. 8 we show some analytical stability thresholds ${ }^{5}$ computed for different values of the total time delay $\mathrm{T}_{\mathrm{d}}$. The curves refer to a phase margin of $60^{\circ}$. The results of the code simulations match the analytical predictions very well.

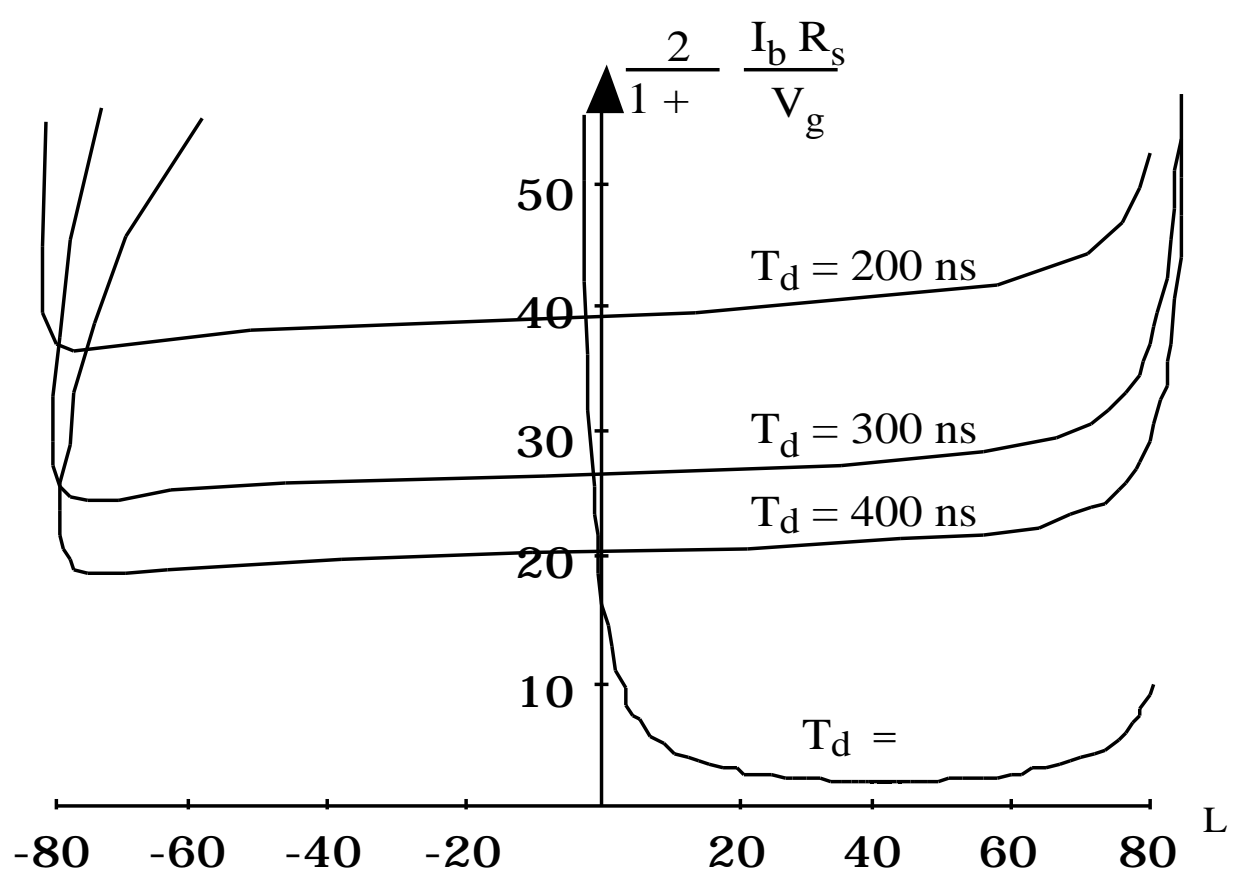

Fig. 8: Robinson's stability limits at different $T_{d}$.

\subsection{Above Robinson's stability limit.}

It is interesting to spend a few words on what happens above the Robinson's stability limit. For given values of the forward voltage $V_{f}$, beam loss voltage $V_{1}$ and cavity fundamental mode impedance $Z_{0}$, below the instability threshold there is only one mathematical solution for the gap accelerating voltage $V_{g}$ and the synchronous phase $\phi_{\mathrm{s}}$. Above the stability limit a second mathematical solution appears corresponding to a higher $\mathrm{V}_{\mathrm{g}}$ value and a new synchronous phase ${ }^{9}$. Even if this solution satisfies the Robinson's stability criterion, in the real operation the automatic RF level control circuit (AGC) prevents the system from approaching this operating condition.

Since the AGC system has not been implemented in the code, above the static Robinson's threshold the beam in the simulations can either get unstable or find a new equilibrium around the new synchronous phase depending on the starting conditions. 


\section{Multibunch simulations.}

We have performed the simulations with all the measured HOMs in the cavity ${ }^{1,2}$, with 29 bunches at their equilibrium position and simulating the injection of the $30^{\text {th }}$ bunch with an error of 100 psec.

The phase oscillations of the injected bunch and of one of the 29 perturbed bunches during the first 5000 turns are shown in Fig. 9. The effects of both RF and bunch-by-bunch feedback systems are included in the simulation and the results are very similar to those obtained in the perfectly compensated case ${ }^{1,2}$.

If we turn off the longitudinal bunch-by-bunch feedback, we observe in Fig. 10 a slow instability growth due to the damped HOMs.
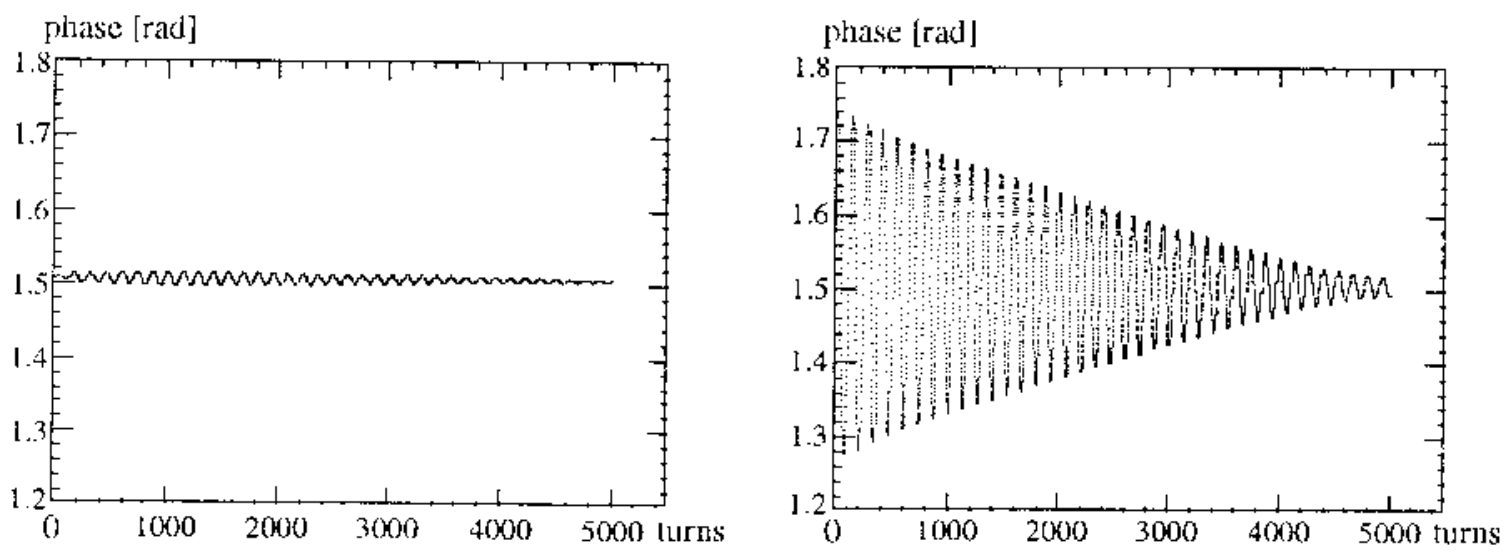

Fig. 9: Oscillations with both longitudinal and RF feedback systems on.
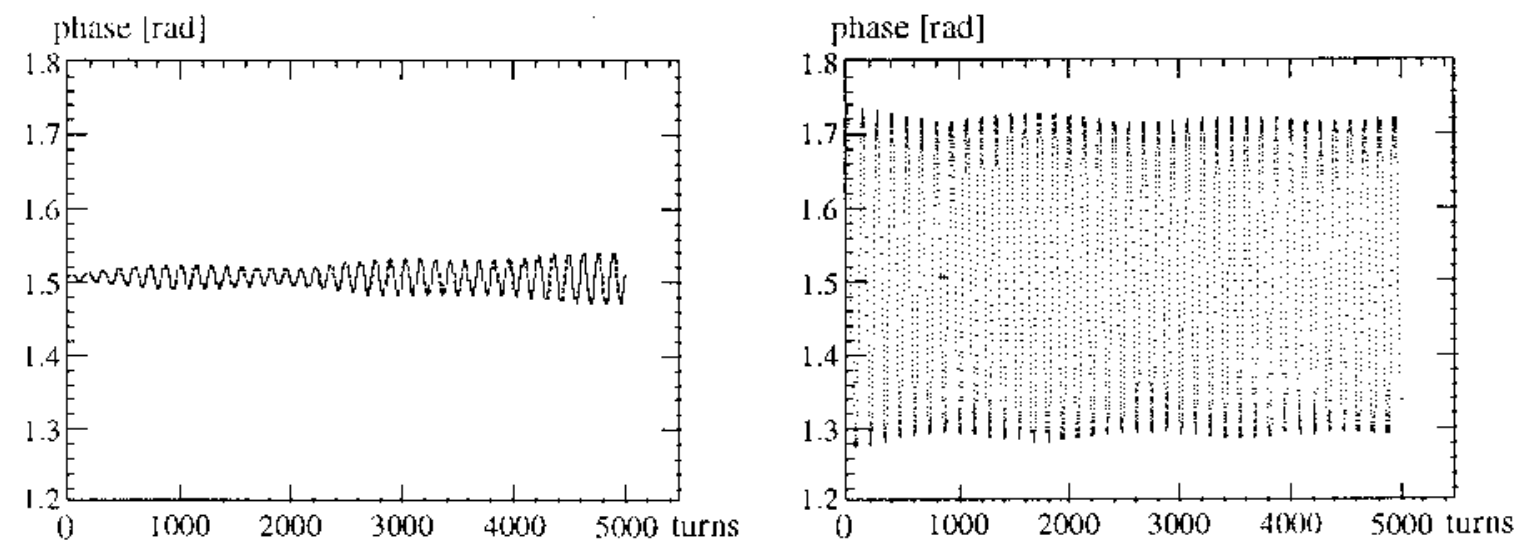

Fig. 10: Oscillations with the longitudinal feedback system off.

Without the RF feedback (Fig. 11) we have instead a very fast instability because we are above the static Robinson's stability limit. In this case the longitudinal feedback can not damp the instability because the bunches do not oscillate at all, but their phase increases exponentially since the longitudinal force becomes defocusing. 


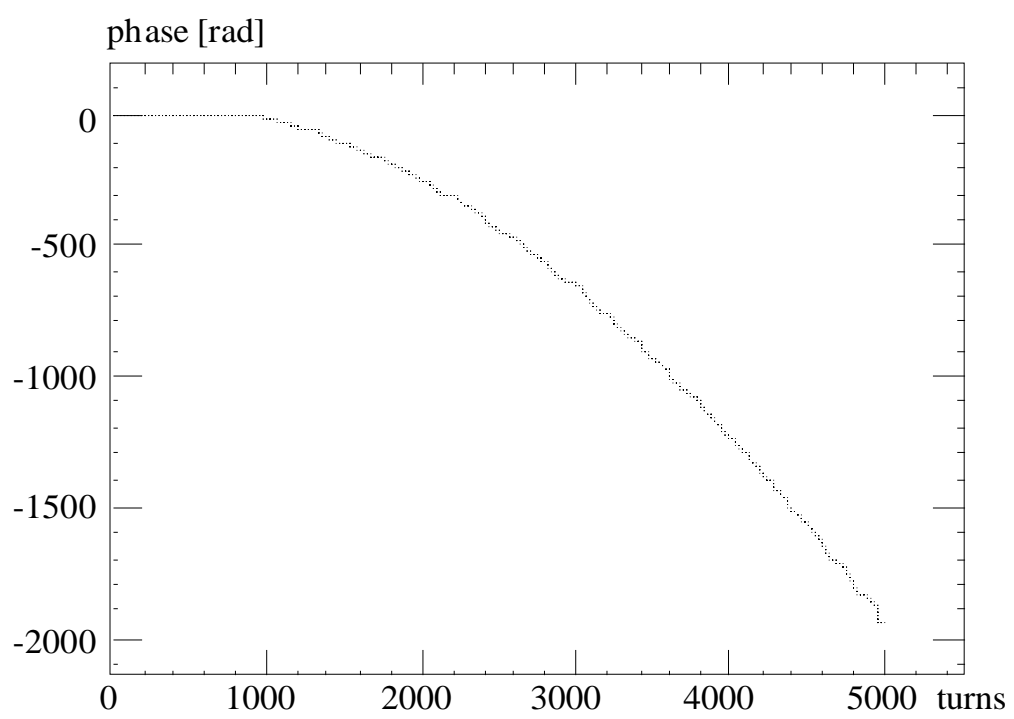

Fig. 11: Bunch instability with the RF feedback system off.

\section{Conclusions.}

The lumped network presented in this note has been adopted to simulate the RF feedback system. It approximates the klystron response and the delay lines of the real system near the cavity resonant frequency $\omega_{\mathrm{c}}$ quite well.

The simulation code reproduces all the instabilities predicted by the theory with great accuracy. The multibunch simulations do not give any new unexpected result but they are in agreement with the theory and show that the feedback system can reduce the effect of the beam loading avoiding the static Robinson's instability. We can also conclude that the multibunch collective dynamics in presence of an efficient RF feedback system can be well analyzed by considering the ideal case of perfectly compensated beam loading of the fundamental mode.

\section{Acknowledgments.}

We benefited very much from the supervision of D. Boussard, R. Boni and L. Palumbo to this work.

\section{REFERENCES}

[1] S. Bartalucci et al., "Analysis of Methods for Controlling Multibunch Instabilities in DAФNE", LNF-93/067(P), October 1993.

[2] M. Bassetti et al., "A Time Domain Simulation Code of the Longitudinal Multibunch Instabilities", DAФNE Technical Note G-19, June 1993.

[3] M. Bassetti et al., "Beam Loading in DAФNE Cavities", Proc. of the $3^{\text {rd }}$ European Particle Accelerator Conference, Berlin March 1992, p. 801.

[4] R. Boni, A. Gallo, "Stationary Beam Loading On DAФNE Cavity Accelerating Mode", DAФNE Technical Note RF-5, February 1992.

[5] A. Gallo, "An RF Feedback for DAФNE", DAФNE Technical Note RF-6, May 1992.

[6] M. Migliorati, L. Verolino, "Stability Analysis and Pulse Response of an RF Feedback System", DAФNE Technical Note RF-9, March 1993.

[7] A. Hofmann, "Single-Beam Collective Phenomena - Longitudinal", CERN 77-13, pp. 139174, Erice 1976.

[8] M. Sands, "Beam-Cavity Interaction I. Basic Considerations", Rapport Technique 2-76, Orsay, June 1976.

[9] M. Migliorati, Dissertation, University of Rome "La Sapienza", A.A. 1990-91, unpublished. 


\section{APPENDIX 1}

From the circuit of Fig. 5, in the Lapalce domain and by neglecting $I_{b}$ and $V_{\text {Ref }}$, it is possible to obtain the following relations:

$$
\left\{\begin{array}{l}
V_{c}(s)=\frac{s P_{1}(s)}{Q(s)} V_{c}(0)-2 \alpha R_{s} \frac{P_{1}(s)}{Q(s)} I_{c}(0)-8 \alpha \beta G \frac{s^{2}}{Q(s)} V_{d}(0)+16 \alpha \alpha^{\prime \prime} \beta G R_{d} \frac{s}{Q(s)} I_{d}(0) \\
I_{c}(s)=\frac{\omega_{c}^{2}}{2 \alpha R_{s}} \frac{P_{1}(s)}{Q(s)} V_{c}(0)+\frac{P_{2}(s)}{Q(s)} I_{c}(0)-\frac{4 \beta G \omega_{c}^{2}}{R_{s}} \frac{s}{Q(s)} V_{d}(0)+\frac{8 \alpha^{\prime \prime} \beta G R_{d} \omega_{c}^{2}}{R_{s}} \frac{1}{Q(s)} I_{d}(0) \\
V_{d}(s)=2 \alpha^{\prime \prime} \frac{s^{2}}{Q(s)} V_{c}(0)-4 \alpha \alpha^{\prime \prime} R_{s} \frac{s}{Q(s)} I_{c}(0)+\frac{s P(s)}{Q(s)} V_{d}(0)-2 \alpha^{\prime \prime} R_{d} \frac{P(s)}{Q(s)} I_{d}(0) \\
I_{d}(s)=\frac{\omega_{d}^{2}}{R_{d}} \frac{s}{Q(s)} V_{c}(0)-\frac{2 \alpha R_{s} \omega_{d}^{2}}{R_{d}} \frac{1}{Q(s)} I_{c}(0)+\frac{\omega_{d}^{2}}{2 \alpha^{\prime \prime} R_{d}} \frac{P(s)}{Q(s)} V_{d}(0)+\frac{P_{3}(s)}{Q(s)} I_{d}(0)
\end{array}\right.
$$

where

$$
\begin{gathered}
\alpha=\frac{1}{2 C_{c} R_{s}}, \quad \alpha^{\prime}=\alpha(1+\beta-2 \beta G), \quad \alpha^{\prime \prime}=\frac{1}{2 C_{d} R_{d}} \\
P(s)=s^{2}+2 \alpha^{\prime} s+\omega_{c}^{2}, \quad P_{1}(s)=s^{2}+2 \alpha^{\prime \prime} s+\omega_{d}^{2} \\
P_{2}(s)=P_{1}(s)\left(s+2 \alpha^{\prime}\right)+16 \alpha \alpha^{\prime \prime} \beta G s, \quad P_{3}(s)=P(s)\left(s+2 \alpha^{\prime \prime}\right)+16 \alpha \alpha^{\prime \prime} \beta G s \\
Q(s)=P(s) P_{1}(s)+16 \alpha \alpha^{\prime \prime} \beta G s^{2}
\end{gathered}
$$

The system of equations can be written in the matrix form:

$$
\mathbf{X}(s)=\mathbf{A}(s) \mathbf{X}(0)
$$


where

$$
\mathbf{X}(s)=\left(\begin{array}{c}
V_{c}(s) \\
I_{c}(s) \\
V_{d}(s) \\
I_{d}(s)
\end{array}\right)
$$

and $\mathbf{A}(\mathbf{s})$ is a $4 \times 4$ matrix.

The response in the time domain is obtained by the inverse Laplace transform of the matrix elements $a_{i j}(s)$, which are of the general form:

$$
a_{i j}(s)=\frac{P_{i j}^{3}(s)}{Q(s)}
$$

Here $P_{i j}^{3}(s)$ is a $3^{\text {rd }}$ order polynomial at most, and $\mathrm{Q}(\mathrm{s})$ is a $4^{\text {th }}$ order polynomial with real coefficients which has two pairs of complex conjugated solutions, and therefore can be written as:

$$
Q(s)=\left(s^{2}-2 \alpha_{1} s+\omega_{1}^{2}\right)\left(s^{2}-2 \alpha_{2} s+\omega_{2}^{2}\right)
$$

The general form of $a_{i j}(t)$ is then:

$$
a_{i j}(t)=e^{\alpha_{1} t}\left[A_{i j} \cos \left(\beta_{1} t\right)+B_{i j} \sin \left(\beta_{1} t\right)\right]+e^{\alpha_{2} t}\left[C_{i j} \cos \left(\beta_{2} t\right)+D_{i j} \sin \left(\beta_{2} t\right)\right]
$$

where

$$
\alpha_{1}^{2}+\beta_{1}^{2}=\omega_{1}^{2} \quad \alpha_{2}^{2}+\beta_{2}^{2}=\omega_{2}^{2}
$$

and $\left(\mathrm{A}_{\mathrm{ij}}, \mathrm{B}_{\mathrm{ij}}, \mathrm{C}_{\mathrm{ij}}, \mathrm{D}_{\mathrm{ij}}\right)$ are easily obtained with the usual technique of partial fractions.

The final time evolution of the free oscillations is therefore obtained from the transport system:

$$
\left(\begin{array}{c}
V_{c}(t) \\
I_{c}(t) \\
V_{d}(t) \\
I_{d}(t)
\end{array}\right)=\left(\begin{array}{llll}
a_{11}(t) & a_{12}(t) & a_{13}(t) & a_{14}(t) \\
a_{21}(t) & a_{22}(t) & a_{23}(t) & a_{24}(t) \\
a_{31}(t) & a_{32}(t) & a_{33}(t) & a_{34}(t) \\
a_{41}(t) & a_{42}(t) & a_{43}(t) & a_{44}(t)
\end{array}\right)\left(\begin{array}{c}
V_{c}(0) \\
I_{c}(0) \\
V_{d}(0) \\
I_{d}(0)
\end{array}\right)
$$

